\title{
Evolution of the electric potential of an insulator under charged particle impact
}

\author{
E. Giglio, S. Guillous, and A. Cassimi \\ Centre de Recherche sur les Ions, les Matériaux et la Photonique (CIMAP), F-14000 Caen, France, EU
}

\author{
H. Q. Zhang \\ School of Nuclear Science and Technology, Lanzhou University, Lanzhou, China
}

G. U. L. Nagy

Institute for Nuclear Research, Hungarian Academy of Sciences (ATOMKI), H-4026 Debrecen, P.O. Box 51, Hungary, EU

\section{K. Tôkési}

Institute for Nuclear Research, Hungarian Academy of Sciences (ATOMKI), H-4026 Debrecen, P.O. Box 51, Hungary, EU and ELI-ALPS, ELI-HU Non-profit Ltd., Dugonics ter 13, H-6720 Szeged, Hungary, EU

(Received 12 July 2016; revised manuscript received 16 December 2016; published 21 March 2017)

\begin{abstract}
Insulating glass capillaries have been shown to lead to ion transmission without any change in either the ion charge state or in the ion kinetic energy. This surprising process has been attributed to a self-organized distribution of charge patches creating the necessary guiding electric potential on the capillary walls. By the use of our original electrometer, it has been possible to measure and monitor simultaneously and in a nondestructive way the electric potential and the transmitted beam intensity during the charging up by an $\mathrm{Ar}^{+}$ion beam. We show that glass microcapillaries can reach potentials higher than $500 \mathrm{~V}$, even in the case of singly charged ions, opening the possibility of high transmission rates and providing a renewed sight into ion beam transport by tapered capillaries. The setup, also suitable for the determination of leakage currents governing the capillary potential dynamics, allowed one to evidence that secondary electrons may strongly affect the rise of the capillary potential and consequently avoid Coulomb blocking of the beam transmission across insulating capillaries.
\end{abstract}

DOI: 10.1103/PhysRevA.95.030702

Under charged particles impact, insulator surfaces accumulate electric charges, generating an electric field that, if sufficiently strong, prevents the following beam particles to hit the insulator surface. This basic property led to the discovery that slow ions can pass through insulator capillaries, keeping their initial charge state even when the geometrical conditions do not allow it [1]. The phenomenon is called charged particle guiding by insulators and it has become an intensively studied field since its discovery [2-25]. The basic concept is the transmission of charged particles through insulating capillaries under incident angles much larger than the geometrical transmission due to the self-organized formation of charge patches at the inner wall that guide the ions. Taking advantage of the existing knowledge with insulating capillaries, a unique experimental technique was developed for the investigation of charging and discharging processes in insulating surfaces. The technique is based on the combination of slow ions and a conical-shape insulator glass capillary and allows following in time the accumulated charge in the capillary with a sensitivity of $20 \mathrm{pC}$. Alternatively, it allows measuring leakage currents in the femtoampere range.

When an ion beam is injected into a tapered glass capillary, most of the injected charge is stored in the dielectric and its electric potential rises. How exactly the potential evolves is difficult to monitor, which may explain why the potential of charged capillaries is hardly mentioned in the literature in the analysis of the transmission of tapered capillaries. Also, the inner resistance of most electrometers is lower or comparable to that of a glass capillary and would spuriously discharge the capillary. However, knowing the transmission rate as a function of the capillary potential, is crucial for the understanding of the charge dynamics in capillaries. For example, it allows one to identify if a possible blocking of the transmission is indeed due to Coulomb blocking, by comparing the potential of the capillary to the extraction potential of the ions. A recent theoretical study showed that the transmission through conical-shaped capillaries increases strongly when the self-organized electric potential approaches about $80 \%$ of the extraction potential of the ion source, as the focal point is moved from infinity to the exit of the capillary [24]. For a low enough beam emittance, transmission rates of a factor 20 well above the geometrical one are expected, boosting the interest of ion beam transport by conical-shaped glass capillaries. Thus, linking the transmission rate to the capillary potential will allow one to identify the critical moment where a significant increase of the intensity can be expected. We present a setup and a technique, able to monitor simultaneously the time evolution of the electric potential and the transmitted beam intensity of a tapered capillary during the charging up, in a nondestructive way. We expect this original technique to be particularly useful to the community of capillaries, as the potential of the capillary was often a missing brick for the interpretation of the results. Meanwhile, this study also evidences that stray electrons can be a strong discharge mechanism that helps to stabilize the electric potential and avoiding Coulomb blocking. Eventually, it permits one to quantify the leakage current due to stray electrons during the charging process.

We use a borosilicate glass microcapillary of $75 \mathrm{~mm}$ length, whose first segment of $35 \mathrm{~mm}$ is cylindrical, while the end was conical shaped. The inner diameter of the outlet of our conical capillary is $26 \mu \mathrm{m}$ and is 33 times smaller than the 


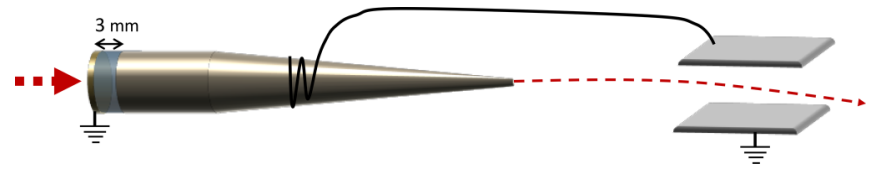

FIG. 1. Electrode setup of conical-shaped borosilicate capillaries with deflection plates. The 3-mm insulator gap of $\sim 10^{15} \Omega$ separates the grounded entrance from the floating electrodes.

inner diameter of the inlet $(860 \mu \mathrm{m})$. The entrance of the capillary, in electric contact with the inlet collimator hole of $600 \mu \mathrm{m}$ diameter, is on ground potential. This results in a geometrical transmission fraction of less than $0.2 \%$. The outer surface of the capillary is covered with a conductive layer (silver paint), except for $3 \mathrm{~mm}$ behind the entrance (see Fig. 1), which acts as a resistor of $(1.1 \pm 0.2) \times 10^{15} \Omega$. The outer metallic surface, of an estimated capacity of $0.9 \mathrm{pF}$, was connected by a thin copper cable of negligible capacity to a plate electrode placed behind the capillary exit, which was acting as a deflector with its grounded pair. The deflector plates have a surface of $9 \mathrm{~cm}^{2}$ and are separated by $1 \mathrm{~cm}$, giving an estimated capacity of about $0.8 \mathrm{pF}$, if ignoring any border effects. The upper (connected) deflection plate is maintained by a resistor of $(7.5 \pm 0.1) \times 10^{14} \Omega$. Both resistances were measured at room temperature and at a pressure below $10^{-6}$ mbar. The conductive layer could be connected to an ammeter or, according to our experimental conditions, kept floating. The experimental chamber is equipped with a high precision rotatable and translational capillary holder and a position sensitive detector (PSD), using a microchannel plate and delay line anode located $400 \mathrm{~mm}$ behind the deflector plates. Inside the holder, the capillary is fixed mechanically. The measurements were performed at the CIMAP laboratory in Caen, France. For the experiments, we used $3 \mathrm{kV} \mathrm{Ar}^{+}$ions produced by an ion gun. For the charging, the intensity of the incoming ion beam was varied between 0.4 and $16 \mathrm{pA}$. The beam divergence is estimated to be less than $0.3^{\circ}$. The capillary axis was aligned with the beam. The injected current $I$ was measured precisely before charging up and after discharging, by connecting the outer electrode to an ammeter. Indeed, in this configuration, the outer metal layer behaves like a Faraday cup.

The main purpose here is to monitor the electric potential of a tapered capillary during the charge-up process in a nondestructive way. The key idea is to keep the potential of the upper deflection plate, connected to the metallized outer capillary surface, floating. The charge-up and the discharge processes are competing. One is the charging of the capillary due to the incoming ion beam of intensity $I$. The other one is the leakage current, controlled by the equivalent resistance $R=(4.4 \pm 1.4) \times 10^{14} \Omega$ of the two resistors (in parallel) between the grounded and floating electrodes. Let $C \simeq 1.7 \mathrm{pF}$ be the capacity of the deflector plates in parallel with the capillary metal surface and $U$ their potential. The equivalent circuit of the setup is given in Fig. 2. We label $\tau_{0}=$ $C R$ the characteristic discharge rate of the capacitor through the resistance $R$. An ion beam of intensity $I$ is injected into the glass capillary and a small fraction of the beam is transmitted through the capillary immediately, while the dominant fraction

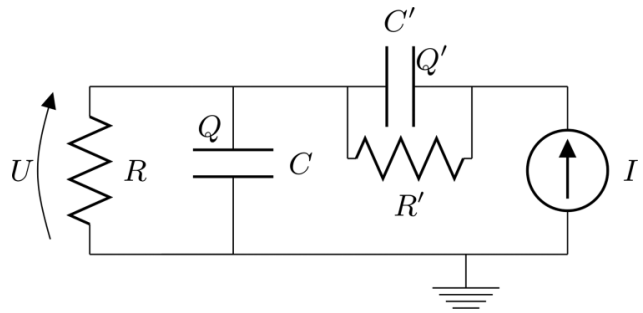

FIG. 2. Equivalent electrical circuit of the electrode setup. The resistance $R$ stands for the resistance separating the floating electrodes of capacity $C$ from the ground. The capacity $C^{\prime}$ and impedance $R^{\prime}$ of the glass-metal interface at the outer capillary surface have been added for completeness, but are irrelevant for this study. They merely control the drop of the potential at the interface.

of the beam hits the inner surface, accumulating the charge on the inner wall. Because of the low geometrical transmission $(<0.2 \%)$, the deposited charge $d Q^{\prime}$ per unit time $d t$ at the inner surface is thus safely approached by $d Q^{\prime}=I d t$. As the outer metal layer covers almost all the capillary, the inner and outer surfaces of the capillary are in total influence. Consequently, the charge stored in the glass is distributed on the electrodes. From the circuit, one deduces that the potential $U$ of the floating outer metallic layer and upper deflection plate rises in time like $U(t)=I R\left[1-\exp \left(-t / \tau_{0}\right)\right]$, generating thus an increasing electric field between the deflector plates. The transmitted ions are deflected by the electric field on a PSD. From the impact position on the detector, we were able to deduce the potential of the target from a previously generated look-up table. Once the incoming beam current and the leakage currents are equal, a stable potential (i.e., a stable deflection) is observed on the detector.

In our chamber, the capillary was initially not shielded from secondary electrons, produced by the ion impacts on metallic parts. As a consequence, the charging dynamics of the capillary was strongly affected by stray electrons, which were captured by the positive potential of the capillary and upper deflector plate, giving rise to a spurious discharge channel. This channel was so strong that the capillary stayed at a low potential. To reduce the neutralization channel due to stray electrons, the capillary holder was wrapped in aluminum foil. We could, however, only achieve a partial shielding. First, the deflection plate was understandingly not shielded and could still attract stray electrons, once its potential rose. Second, stray electrons could still slip through the 1-mm gap below the collimator plate, bypassing the shielding. Nevertheless, after the partial shielding, the discharge rate of the channel was decreased strongly, allowing finally the rise of the capillary potential. The injected secondary electrons current at the outer metallized capillary surface now no longer exceeded the injected beam current $I$, while still dominating the extremely low leakage current of an electrically isolated capillary. Eventually this permitted us to evidence the sensitivity of the capillary potential to stray electron neutralization.

As the secondary electrons are not fully suppressed, we add a depletion channel accounting for the secondary electrons that are injected at the positive but floating electrodes. The injected electron current is estimated using the following assumptions: First, we assume that the secondary electrons that are possibly 
injected at the electrodes, bypassing the incomplete shielding, are mostly produced by the beam ions that hit the collimator plate in front of the capillary. This electron current is thus taken proportional to the injected ion beam current $I$, with $k>0$ being the proportionality factor, depending mainly on the screening. Second, we assume that with increasing potential, more and more electrons are attracted toward the electrodes. We assume that its probability increases linearly for low potentials and that there exists a threshold potential $U_{c}$ where all available electrons are injected at the electrodes. The probability that satisfies both requirements may be expressed by an error function, $0 \leqslant \operatorname{erf}\left(U / U_{c}\right)<1$, and the time evolution of $U$ is eventually given by

$$
C \frac{\partial U}{\partial t}=I-\frac{U}{R}-k I \operatorname{erf}\left(U / U_{c}\right)
$$

The solution of the above equation may be obtained analytically for the two cases where $U \ll U_{c}$ and $U \gg U_{c}$. If the potential $U$ is sufficiently low compared to $U_{c}, k \operatorname{erf}\left(U / U_{c}\right) \simeq$ $\frac{2 k}{\sqrt{\pi} U_{c}} U=\alpha U$, then Eq. (1) resumes to

$$
\frac{\partial U}{\partial t}=\frac{I}{C}-\left(\frac{1}{\tau_{0}}+\frac{\alpha I}{C}\right) U .
$$

Its solution is given by

$$
U(t)=U_{0} e^{-t / \tau}+\frac{I \tau}{C}\left(1-e^{-t / \tau}\right),
$$

where we define the characteristic time scale $\tau$ and the initial potential by $U_{0}$,

$$
\tau \equiv \tau(I)=\frac{\tau_{0}}{1+\alpha R I} \quad \text { and } \quad U_{0}=U(t=0) .
$$

Equation (3) fits both charging and discharging of the capillary in the presence of secondary electrons. Within these assumptions, the asymptotic potential that is reached during charge-up,

$$
U(t \rightarrow+\infty, I)=\frac{R I}{1+\alpha R I},
$$

increases with the input current $I$. However, for larger input currents, the assumption that $U$ is small compared to $U_{c}$ may no longer be valid. The probability $\operatorname{erf}\left(U / U_{c}\right)$ tends asymptotically to 1 when $U$ is larger than $U_{c}$ and Eq. (1) resumes to

$$
\frac{\partial U}{\partial t}=\frac{I(1-k)}{C}-\frac{U}{\tau_{0}} .
$$

Its solution is $U(t)=R I(1-k)\left(1-e^{-t / \tau_{0}}\right)+U_{0} e^{-t / \tau_{0}}$. In this case, the asymptotic potential, which is reached during charge-up, now becomes

$$
U(t \rightarrow+\infty, I)=R I(1-k) .
$$

It increases linearly with the beam intensity $I$, where the slope depends on $k$, which is essentially the screening of the electrodes from the secondary electrons. In the case of very low input currents $I \rightarrow 0$, Eq. (4) shows that $\tau(I)$ tends asymptotically to the characteristic time $\tau_{0}$, from which the resistance $R$ or capacity $C$ may be deduced, if one of them is known.

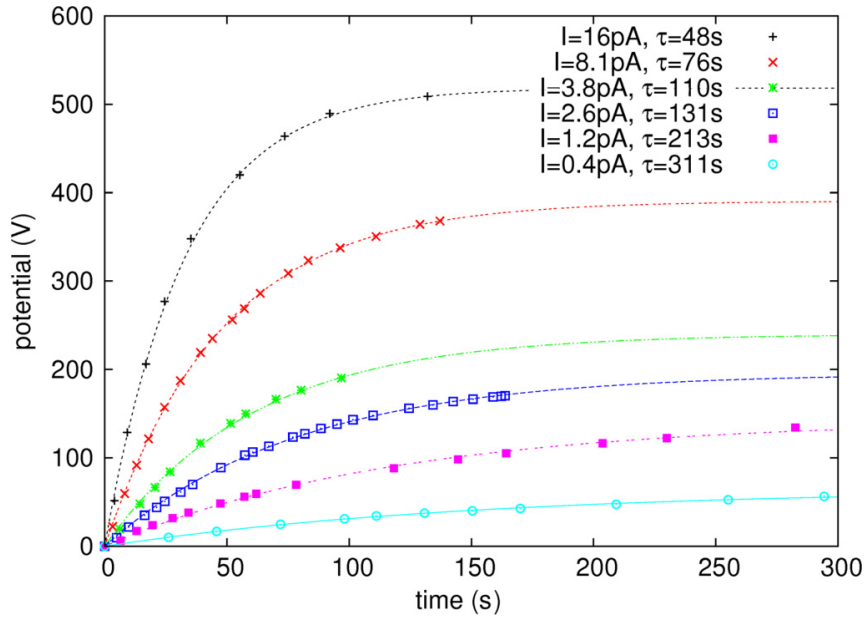

FIG. 3. Monitoring of the potential of the capillary during the charging with a constant beam intensity as indicated in the legend. The characteristic time constants have been extracted from the fit of the experimental data with Eq. (3).

Charging measurements where done for several injected beam intensities. In each case, the capillary was initially discharged, $U_{0}=0$, and the outer metal layer was connected to an ammeter to measure the injected beam intensity for about $20 \mathrm{~s}$. Then, the metal layer was disconnected from the ammeter and kept floating. The geometrically transmitted part of the beam passes through the deflection plates and reaches the PSD after $400 \mathrm{~mm}$ of free fly. The deflection of the beam is recorded as a function of time. From a calibration look-up table, linking the position on the PSD to the potential of the electrodes, we obtain the time evolution of the potential, shown in Fig. 3. The experimental data are then fitted by Eq. (3), from which the characteristic times $\tau$ and asymptotic potentials are extracted. Note that the characteristic times decrease quickly with the beam intensity, indicating clearly that the depletion channel due to secondary electrons dominates the leakage current through the resistance $R$. With our setup, we reached potentials up to $500 \mathrm{~V}$, which is still well below the threshold value of $2400 \mathrm{~V}$ ( $80 \%$ of the extraction potential) by which the capillary may start to focus the beam and significantly increase the transmitted beam intensity. As a result, the transmission during the charging always remained close to the optical transmission of $0.2 \%$. The asymptotic value of the potential of the electrodes increases monotonously with increasing beam current, as shown in Fig. 4. The low intensity part of the curve is well fitted by expression (5). The high intensity part of the curve increases linearly as predicted by our model. From both fits, the constant $\alpha^{-1}=228 \pm 14 \mathrm{~V}$ and the slope $R(1-k)=(20 \pm 2) \times 10^{12} \Omega$ are extracted from Fig. 4. Using the definition of $\alpha$, one obtains $U_{c}=245 \pm$ $17 \mathrm{~V}$. This means that in our setup, the screening was such that for a capillary potential above $245 \mathrm{~V}$, all of the available electrons, which are produced at the collimator and at the detector and bypass the shielding, are injected at the electrodes. Finally, we can calculate $k=0.95 \pm 0.01$, indicating that the injected positive current into the capillary is almost compensated by the injected electrons at the outer electrodes. Nevertheless, the linear trend shown in Fig. 4 indicates that 


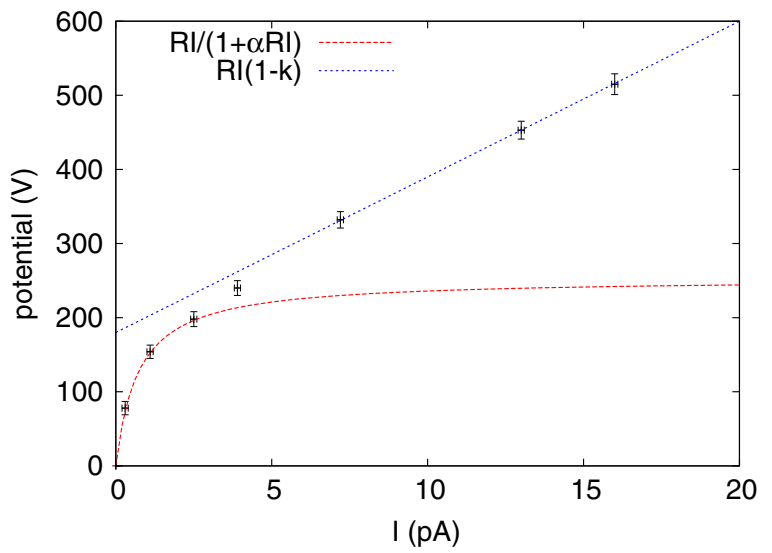

FIG. 4. Asymptotic potential reached during the charge-up (black points) as a function of the injected beam intensity. The low intensity part of the curve is well fitted by expression (5). Above the threshold $U_{c}=245 \mathrm{~V}$, the curve increases linearly as predicted by Eq. (7). The uncertainties on the potentials are those given by the fit in Fig. 3. An absolute uncertainty of $0.1 \mathrm{pA}$ is estimated on the currents.

conical glass capillaries may reach potentials approaching the $\mathrm{kV}$ range and be suitable candidates for focusing ion beams and achieve high transmission rates, well above the geometrical one. This feature may be exploited, for example, in ion beam lines.

While secondary electrons indeed limit the asymptotic potential that a nude tapered capillary can reach for a given input beam current, they also help to avoid Coulomb blocking of the capillary by keeping the potential below the extraction potential of the beam source. We expect that this phenomenon is responsible for the nonblocking of the tapered glass capillary in the measurements shown by $\mathrm{M}$. Kreller et al. [15]. They showed that over $20 \mathrm{nC}$ were injected into a conical borosilicate capillary of estimated capacity of $1 \mathrm{pF}$, using a $80 \mathrm{pA}, \mathrm{Ar}^{8+}$ beam at $24 \mathrm{keV}$ for over $4 \mathrm{~min}$. If there were no additional depletion channel, then due to resistance of the insulating capillary, the capillary would have exceeded the extraction potential of $600 \mathrm{~V}$ already after $10 \mathrm{~s}$ of beam time. Similarly, the spuriously fast characteristic discharge time of 4.4 min for a borosilicate capillary, as measured by Cassimi et al. [17], is expected to be due to the lack of screening of the capillary from secondary electrons. To a certain extent, the effect of the additional discharge channel due to secondary electrons is similar to increasing the conductivity of the capillary by increasing its temperature $[12,16]$.

Once the asymptotic deflection was attained, the discharge was initiated by reducing the injected beam intensity $I$ to about $10 \mathrm{fA}$. For such small injected intensities, the leakage current $U / R$ through the resistance $R$ dominates the injected beam current as well as the neutralization channel due to stray electrons. From the discharge curve we extract the time constant $\tau=600 \mathrm{~s}$ (not shown), which is a good estimate for the characteristic time constant $\tau_{0}=R C$ of the setup. Taking $R=(4.4 \pm 1.4) \times 10^{14} \Omega$, the capacity of the electrodes becomes $(1.4 \pm 0.4) \mathrm{pF}$, in relative good agreement with the estimated capacity of about $C \simeq 1.7 \mathrm{pF}$.

In conclusion, we used the deflection of the transmitted beam as an ideal electrometer to monitor the potential of an insulating surface, which in our recent case was the inner surface of the tapered glass capillary, under charged particle impacts as a function of time. Simultaneously we recorded the transmitted beam intensity. Measuring the potential of the capillary while following the transmitted beam intensity is of great interest for the community of capillaries. It may indeed be helpful to identify if the blocking of the transmission is due to Coulomb blocking or if the blocking happened because, for example, the beam is deviated away from the exit by charge patches due to the mismatch of the capillary axis with the beam axis or the asymmetries of the capillary. The technique is also helpful for identifying if and when the capillary reaches the critical potential where it starts to act as an electrostatic lens, opening a pathway to control the transmission rate by the change of the capillary potential, in a self-organized manner. Our measurements show that, despite the neutralization channel due to stray electrons, a tapered glass capillary is able to accumulate enough charge to generate electric potentials that go beyond $600 \mathrm{~V}$. This is encouraging for the study of the lens effect of capillaries as, with a better screening, we expect the potential to easily access the kilovolt range. Last but not least, with the help of a simple model, we were able not only to identify but also to quantify the leakage currents during the capillary charge-up. We clearly show that, if the capillary is not sufficiently screened from secondary electron sources, stray electrons hinder the potential of the capillary to approach the extraction potential of the ions. However, this secondary electron channel might have allowed other authors to inject strong beam currents into tapered glass capillaries without experiencing Coulomb blocking. Finally, this technique may be used to evaluate the screening of the capillary from stray electrons in experimental setups.

This work was funded by the French National Research Agency (ANR) in the P2N 2012 program (ANR-12-NANO008) for the PELIICAEN project. This work was also supported by TéT Grant No. TéT_12_FR-1-013-0007, by Hungarian Scientific Research Fund OTKA No. NN 103279, and by the European Cost Actions CM1204 (XLIC) and CM1405 (MOLIM). H.Z. acknowledges the financial support by the National Natural Science Foundation of China (Grant No. 11475075).
[1] N. Stolterfoht, J.-H. Bremer, V. Hoffmann, R. Hellhammer, D. Fink, A. Petrov, and B. Sulik, Phys. Rev. Lett. 88, 133201 (2002).

[2] K. Schiessl, W. Palfinger, K. Tőkési, H. Nowotny, C. Lemell, and J. Burgdörfer, Phys. Rev. A. 72, 062902 (2005).
[3] T. Ikeda, Y. Kanai, T. M. Kojima, Y. Iwai, T. Kambara, and Y. Yamazaki, M. Hoshino, T. Nebiki, and T. Narusawa, Appl. Phys. Lett. 89, 163502 (2006).

[4] M. B. Sahana, P. Skog, G. Vikor, R. T. Rajendra Kumar, and R. Schuch, Phys. Rev. A 73, 040901(R) (2006). 
[5] A. R. Milosavljević, Gy. Víkor, Z. D. Pešić, P. Kolarž, D. Šević, B. P. Marinković, S. Mátéfi-Tempfli, M. Mátéfi-Tempfli, and L. Piraux, Phys. Rev. A 75, 030901(R) (2007).

[6] P. Skog, H. Q. Zhang, and R. Schuch, Phys. Rev. Lett. 101, 223202 (2008).

[7] A. Cassimi, T. Muranaka, L. Maunoury, H. Lebius, B. Manil, B. A. Huber, T. Ikeda, Y. Kanai, T. M. Kojima, Y. Iwai, T. Kambara, Y. Yamazaki, T. Nebiki, and T. Narusawa, Int. J. Nanotechnol. 5, 809 (2008).

[8] R. J. Bereczky, G. Kowarik, F. Aumayr, and K. Tókési, Nucl. Instrum. Methods Phys. Res., Sect. B 267, 317 (2009).

[9] Y. Kanai, M. Hoshino, T. Kambara, T. Ikeda, R. Hellhammer, N. Stolterfoht, and Y. Yamazaki, Phys. Rev. A 79, 012711 (2009).

[10] A. Cassimi, L. Maunoury, T. Muranaka, B. Huber, K. R. Dey, H. Lebius, D. Lelievre, J. M. Ramillon, T. Been, T. Ikeda, Y. Kanai, T. M. Kojima, Y. Iwai, Y. Yamazaki, H. Khemliche, N. Bundaleski, and P. Roncin, Nucl. Instrum. Methods Phys. Res., Sect. B 267, 674 (2009).

[11] K. Schiessl, K. Tókési, B. Solleder, C. Lemell, and J. Burgdörfer, Phys. Rev. Lett. 102, 163201 (2009).

[12] N. Bundaleski, H. Khemliche, P. Rousseau, A. Cassimi, L. Maunoury, and P. Roncin, J. Phys.: Conf. Ser. 163, 012091 (2009).

[13] R. G. Kowarik, R. J. Bereczky, F. Aumayr, and K. Tőkési, Nucl. Instrum. Methods Phys. Res., Sect. B 267, 2277 (2009).
[14] B. S. Dassanayake, S. Das, R. J. Bereczky, K. Tókési, and J. A. Tanis, Phys. Rev. A 81, 020701(R) (2010).

[15] M. Kreller, G. Zschornack, and U. Kentsch, Nucl. Instrum. Methods Phys. Res., Sect. B 269, 1032 (2011).

[16] E. Gruber, G. Kowarik, F. Ladinig, J. P. Waclawek, D. Schrempf, F. Aumayr, R. J. Bereczky, K. Tőkési, P. Gunacker, T. Schweigler, C. Lemell, and J. Burgdörfer, Phys. Rev. A 86, 062901 (2012).

[17] A. Cassimi, T. Ikeda, L. Maunoury, C. L. Zhou, S. Guillous, A. Mery, H. Lebius, A. Benyagoub, C. Grygiel, H. Khemliche, P. Roncin, H. Merabet, and J. A. Tanis, Phys. Rev. A 86, 062902 (2012).

[18] R. D. Dubois and K. Tókési, Nucl. Instrum. Methods Phys. Res., Sect. B 279, 186 (2012).

[19] K. Tókési, I. Rajta, R. J. Bereczky, and K. Vad, Nucl. Instrum. Methods Phys. Res., Sect. B 279, 173 (2012).

[20] C. Lemell, J. Burgdörfer, and F. Aumayr, Prog. Surf. Sci. 88, 237 (2013).

[21] N. Stolterfoht, Phys. Rev. A 87, 012902 (2013).

[22] N. Stolterfoht, Phys. Rev. A 87, 032901 (2013).

[23] G. U. L. Nagy, I. Rajta, R. J. Bereczky, and K. Tőkési, in Experimental setup for studying guiding of proton microbeam, AIP Conf. Proc. No. 1525 (AIP, Melville, NY, 2013), p. 40.

[24] E. Giglio, R. D. Dubois, A. Cassimi, and K. Tókési, Nucl. Instrum. Methods Phys. Res., Sect. B 354, 82 (2015).

[25] N. Stolterfoht and Y. Yamazaki, Phys. Rep. 629, 1 (2016). 